\title{
Expanding Treatment Access for Chronic Hepatitis C with Task-shifting in the Era of Direct-acting Antivirals
}

\author{
Eric R. Yoo ${ }^{1}$, Ryan B. Perumpail ${ }^{2}$, George Cholankeril ${ }^{3}$, Channa R. Jayasekera ${ }^{2,4}$ \\ and Aijaz Ahmed*2 \\ ${ }^{1}$ Department of Medicine, University of Illinois College of Medicine, Chicago, IL, USA; ${ }^{2}$ Division of Gastroenterology and \\ Hepatology, Stanford University School of Medicine, Stanford, CA, USA; ${ }^{3}$ Division of Gastroenterology and Hepatology, University \\ of Tennessee Health Sciences Center, Memphis, TN, USA; ${ }^{4}$ Center for Innovation in Global Health, Stanford University, \\ Stanford, CA, USA
}

\begin{abstract}
In the United States, the fight to eradicate hepatitis $C$ virus (HCV) infection has been ongoing for many years, but the results have been less than ideal. Historically, patients with chronic hepatitis $\mathrm{C}(\mathrm{CHC})$ were treated with interferon-based regimens, which were associated with frequent adverse effects, suboptimal response rates, and long durations of treatment - of up to 48 weeks. Expertise from specialistphysicians, such as hepatologists and gastroenterologists, was needed to closely follow patients on these medications so as to monitor laboratory values and manage adverse effects. However, the emergence of direct-acting antiviral (DAA) agents against $\mathrm{HCV}$ infection have heralded outstanding progress in terms of safety, tolerability, lack of adverse effects, efficacy, and truncated duration of therapy - 12 weeks or less - thereby making the need for close monitoring by specialist-physicians obsolete. With the recent approval of DAA agents by the Food and Drug Administration, the treatment model for $\mathrm{CHC}$ no longer relies on the limited number of specialist-physicians, which represented a major barrier to treatment access in the past, especially in underserved areas of the United States. We propose and share our experiences in adapting a task-shifting treatment model, one that utilizes a relatively larger pool of non-specialist healthcare providers, such as nursing staff (medical assistants, vocational licensed nurses, registered nurses, etc.) and advanced practice providers (nurse practitioners and physician assistants), to perform a variety of important clinical functions in an effort to make DAA-based antiviral therapy widely available against $\mathrm{HCV}$ infection. Most recently, task-shifting was implemented by the United States and World Health Organization in the fight against the human immunodeficiency virus and showed
\end{abstract}

Keywords: Task-shifting; Treatment; Access; Underserved; Under-resourced; Hepatitis C virus; HCV; Direct acting antivirals; DAAs.

Abbreviations: $\mathrm{HCV}$, hepatitis $\mathrm{C}$ virus; US, United States; $\mathrm{CHC}$, chronic hepatitis $\mathrm{C}$; CDC, Centers for Disease Control and Prevention; SVR, sustained virological response; PEG-IFN, pegylated-interferon-alpha; RVR, rapid virological response; IFN, interferon; DAA, direct-acting antiviral; FDA, Food and Drug Administration; HIV, human immunodeficiency virus; LVN, licensed vocational nurse; LPN, licensed practical nurse.

Received: 5 November 2016; Revised: 10 February 2017; Accepted: 26 February 2017

*Correspondence to: Aijaz Ahmed, Division of Gastroenterology and Hepatology, Stanford University School of Medicine, 750 Welch Road, Suite \#210, Stanford, CA 94304, USA. Tel: +1-650-498-5691, Fax: +1-650-498-5692, E-mail: aijazahmed@stanford.edu encouraging results. Based on our experiences in implementing this model at our outreach clinics, the majority of HCVinfected patients treated with DAA agents can be easily monitored by non-specialist healthcare providers and physician extenders. Task-shifting can effectively address one of the major rate-limiting factors in expanding treatment access for HCV infection.

Citation of this article: Yoo ER, Perumpail RB, Cholankeril G, Jayasekera CR, Ahmed A. Expanding treatment access for chronic hepatitis $\mathrm{C}$ with task-shifting in the era of directacting antivirals. J Clin Transl Hepatol 2017;5(2):130-133. doi: $10.14218 /$ JCTH.2016.00059.

\section{Introduction}

The hepatitis C virus (HCV) is a single-stranded, positivesense RNA virus that affects over 180 million individuals globally, or $2-3 \%$ of the world's population. ${ }^{1}$ There are seven major genotypes of HCV (genotypes 1-7), and the prevalence of each of these genotypes varies by region, with HCV genotype $1 \mathrm{a}$ having the greatest presence in North America. ${ }^{2}$ Genotype 3 is more difficult to treat and associated with poor prognosis, ${ }^{3}$ and treatment of HCV varies partly due to its error prone viral RNA polymerase and the consequential development of multiple quasispecies within an individual infected with HCV.

In the United States (US), the estimated number of patients with chronic hepatitis $\mathrm{C}(\mathrm{CHC})$ is over 2.5 million and incidence is 30,500 per year; ${ }^{4}$ however, the actual number of those affected is likely higher because a significant proportion of those with $\mathrm{CHC}$ are asymptomatic, with an estimated $45-85 \%$ being unaware of their infection. ${ }^{5}$ Up to $75 \%$ of HCV-infected patients in the US belong to the 'Baby Boomer' birth cohort (birth years 1945 to 1965) and may present with complications of cirrhosis as their initial clinical manifestation. ${ }^{5}$ Not surprisingly, HCV-related end-stage liver disease, with and without hepatocellular carcinoma, is the most common etiology for adult liver transplantation in adults in the US. ${ }^{6}$ In addition, $\mathrm{CHC}$ is also associated with a poor quality of life, significant morbidity, and increase in liverrelated mortality and all-cause mortalities, ${ }^{7,8}$ especially in individuals with advanced liver disease, and accounts for about 10,000 deaths per year in the US. ${ }^{9}$ 
Yoo E.R. et al: Task-shifting for chronic hepatitis C

\section{History of interferon (IFN)-based HCV treatment}

In the US, the battle against $\mathrm{HCV}$ and efforts to reduce $\mathrm{CHC}$ have been ongoing for many years, but have yielded less than ideal results. In 1998, the Centers for Disease Control and Prevention (CDC) created risk-based screening recommendations, which were updated 4 years later to address their limited success. In 2012, the CDC published an update to include one-time screening, regardless of risk, for all individuals born between 1945 and 1965 - the Baby Boomer cohort - because this cohort was found to have significantly higher prevalence compared to other birth cohorts. ${ }^{5}$ Despite efforts to update screening guidelines for $\mathrm{HCV}$ infection, reports have shown that screening is still inadequate and that even after individuals are identified as being anti-HCV positive, follow-up for confirmation and linkage to care is inadequate. ${ }^{10}$ In regards to the treatment of $\mathrm{HCV}$, the goal was, and still is, to eradicate HCV RNA and achieve sustained virological response (SVR), which is defined by the absence of HCV RNA 12 weeks post-treatment. Historically, pegylatedinterferon-alpha (PEG-IFN) and ribavirin were used to treat patients with $\mathrm{CHC}$, with rapid virological response (RVR) being the best predictor of SVR. ${ }^{11}$ While PEG-IFN and ribavirin were used in the past, the duration of treatment traditionally lasted up to 48 weeks, with low efficacy and significant adverse effects. Naturally, treatment access was restricted due to a limited number of hepatologists, followed by gastroenterologists and infectious diseases experts available as well as those well-versed with the interferon-based regimens.

\section{DAAs versus IFN-based regimens}

In adults with $\mathrm{CHC}$, IFN-based regimens have been outclassed by DAA agents, which target viral enzymes responsible for various parts of the HCV life cycle. DAA agents target non-structural proteins and are classified as NS3/4A protease inhibitors, NS5A inhibitors, or NS5B polymerase inhibitors. The recent development of second-generation DAA agents has shown remarkable progress in terms of safety, tolerability, efficacy, duration of therapy, and adverse effect profiles. On June 28, 2016, the US Food and Drug Administration (FDA) approved sofosbuvir/velpatasvir, a fixed-dose DAA combination tablet with a pan-genotypic therapy, to treat all seven major forms of HCV in adult patients with $\mathrm{CHC}$, both with and without cirrhosis. This DAA regimen targets all HCV genotypes, eliminating the need for genotype testing prior to initiating treatment, which was the standard approach with interferon-based regimens. In HCV-infected patients with cirrhosis, sofosbuvir/velpatasvir was approved for use in combination with the drug ribavirin. ${ }^{12}$

\section{Task-shifting treatment model for HCV}

The adaptation of our healthcare system to rapidly emerging advancements in the treatment of $\mathrm{CHC}$ has been rather slow. The safety profile of second-generation DAA agents effectively free patients with $\mathrm{CHC}$ from the historical need for close follow-up with specialist-physicians, who are mostly concentrated in urban areas in the US and who are significantly fewer in number when compared to primary care physicians and supporting nursing staff. There is a more pronounced shortage of specialist-physicians in rural areas of the US.

In an era when every HCV-infected patient is a potential candidate for DAA-based therapy, shortage of providers remains the limiting factor to treatment access. We propose task-shifting as a potential solution to the shortage of healthcare providers with expertise in treatment of $\mathrm{CHC}$. Improved safety, tolerability and efficacy of DAA agents compared to IFN plus ribavirin combination make this approach even more pragmatic. The task-shifting treatment model utilizes a relatively larger pool of non-specialist healthcare providers, such as nursing staff (medical assistants, vocational licensed nurses, registered nurses, etc.) and advanced practice providers (nurse practitioners and physician assistants).

Task-shifting was successfully implemented on a wide scale worldwide in the fight against the human immunodeficiency virus (HIV). Studies have shown that the quality of care in the treatment of HIV infection by non-physicians compared to physicians was comparable in both wellresourced and under-resourced settings. ${ }^{13,14}$ Moreover, the World Health Organization's task-shifting guidelines for HIV allow non-physician providers to perform a variety of functions including, but not limited to, staging HIV, initiating antiretroviral treatment, managing opportunistic infections, interpreting laboratory results, and managing medication complications. ${ }^{15}$

The application of task-shifting the treatment of $\mathrm{CHC}$ may be less complex than that of task-shifting the treatment of HIV infection - the duration of DAA-based treatment for $\mathrm{CHC}$ varies between 8-24 weeks versus indefinite treatment for $\mathrm{HIV}$ infection. In addition, safety profile and adherence (lower pill count and shorter treatment duration) is better for DAA agents compared to antiretroviral therapy for HIV infection. The one potential barrier to a widespread application of taskshifting for the use of DAA agents with non-specialist healthcare providers is the cost of treatment. As more companies develop competing DAA agents, however, treatment will become more affordable.

\section{Other treatment models for HCV treatment}

The task-shifting treatment model is not the only approach to improve access to HCV treatment. Project Extension for Community Healthcare Outcomes (Project ECHO) is a telemedicine and distance-learning model created by Arora et al. to improve treatment access for HCV-infected patients in rural New Mexico. ${ }^{16,17}$ It aims to reduce the need for specialist referrals by using video-conferencing (telemedicine) to connect specialist physicians, such as hepatologists and infectious disease experts, with primary care physicians/providers who are based in rural areas and prisons. On a weekly basis, the community health physicians present their patients to specialists at the nearest academic center, the University of New Mexico (UNM), through telemedicine; medical history, laboratory result(s) and treatment plan are discussed to provide medical management based on best practices. Patients with HCV who were managed through the ECHO model had comparable SVR versus those treated at UNM.

In 2015, the University of Missouri-Columbia also adapted the ECHO model (renamed to Show Me ECHO) to train and mentor primary care physicians treating patients with $\mathrm{HCV}$ in rural and underserved areas of Missouri; ${ }^{18}$ this report subjectively stated the benefits of utilizing the ECHO model ("increased the number of [primary care physicians] who are confident in their ability to diagnose and treat HCVinfected patients," and "increased number of persons detected with [and treated for] HCV...in the targeted geographical areas of the project"). The ECHO model is a 
promising alternative approach in the era of DAA agents to minimize specialist referrals by educating primary care physicians who manage patients with HCV in rural areas and in prisons via weekly video-conferencing (i.e. telemedicine).

This model does allow specialists to stay at their academic centers and manage more complicated patients with HCV infection, including those with cirrhosis and following liver transplantation. The ECHO model may not be a standalone solution to HCV disease burden; however, the role of primary care physicians and video-conferencing technology may be integrated or used alongside the task-shifting model to increase overall access to care.

\section{Our experience with task-shifting through a licensed vocational nurse (LVN)}

Our institution operates three hepatology outreach clinics in medically underserved areas of California, each of which are located between 180 and 230 miles from our main medical center. We adopted, implemented and modified a taskshifting treatment model between 2014 and 2015, treating over 100 patients with $\mathrm{CHC}$ using DAA agents with the help of an LVN, more commonly known as a licensed practical nurse (LPN). Our LVN served as our non-physician healthcare provider, although the task-shifting treatment model can be applied generally to other non-physician providers such as other nursing staff or advanced practice providers.

In our practice, the LVN's tasks included: reviewing symptom calls and laboratory test results with a hepatologist, documenting any changes in management plan, and updating patients through a secured Web portal that allows for access to health information. An index patient was seen and examined by the hepatologist at the time of decision to start antiviral therapy, when a detailed face-to-face discussion was conducted. Subsequently, these patients were only seen if there were any adverse effects reported by the patient. The majority of patients did not require a follow-up visit during the DAA-based therapy and returned for their next follow-up clinic visit 12 weeks after completion of antiviral therapy. The hepatologist (not LVN) conducted the outreach clinic up to three times per month. During the scheduled outreach clinics, laboratory tests and imaging studies performed on index patients undergoing antiviral therapy were reviewed and patients were updated by an LVN via a secured Web portal or by telephone. CHC patients with advanced fibrosis/cirrhosis underwent surveillance for hepatocellular carcinoma every 6 months with alpha-fetoprotein test and ultrasound of the abdomen.

Providers can utilize the electronic medical record to access health data remotely with secure messaging capabilities. Patients also have various ways through which they can stay informed and connected with their providers. Specifically, they may utilize an assigned and personalized account on the secured Web portal to access their health information or directly contact the hepatologist, LVN, or clinic with questions or concerns. Patients needing urgent evaluation are immediately referred to the nearest emergency department, local primary care physician or specialist-physician. For patients with $\mathrm{CHC}$ who are unable to access the Web, we communicate all relevant clinical information via different methods (phone, fax, text and paging), and patients can continue to utilize these tools to communicate effectively with the hepatologist, non-specialist healthcare providers, or the local emergency room, if necessary.
Since the implementation of task-shifting treatment model in 2014, we treated over $100 \mathrm{HCV}$-infected patients with DAAbased treatments through the end of 2015 and have not experienced any DAA-related emergencies. We experienced a few instances of symptoms deemed urgent by patients and a few non-critical laboratory abnormalities, but none of these events warranted a referral to a local urgent care facility or emergency room. Symptom calls necessitating follow-up clinic visits by the hepatologist were noted in less than $5 \%$ of patients and were found to be unrelated to the DAA regimen, with the exception of one case of anemia related to ribavirin use. Up to $20 \%$ of patients reported mild fatigue and self-limited headaches. Our LVN was able to monitor and guide the majority of patients in consultation with the hepatologist without a follow-up clinic visit during the 12- to 24-week course of DAA-based treatment. Our treatment response rates (SVR in particular) were comparable to registration trials.

\section{Primary care providers and task-shifting model for HCV treatment}

As mentioned previously, the task-shifting treatment model can utilize non-specialist healthcare providers such as nursing staff (medical assistants, vocational licensed nurses, registered nurses, etc.) and advanced practice providers (nurse practitioners and physician assistants) with the goal of improving access to HCV treatment in under-resourced rural areas. Primary care physicians practicing in these underserved areas of the US may be better suited to embrace the role of HCV treaters. And, as technology advances and infrastructure for communications in the US continues to improve, addition of videoconferencing/telemedicine can further improve the taskshifting treatment model. On a global scale, and particularly in the Asia-Pacific regions, tackling HCV requires a multifaceted approach for a number of reasons, including continued dependence on physicians to manage $\mathrm{CHC}$, variation in healthcare systems from one nation to the other, and availability/cost of DAA-based treatment. ${ }^{19}$

\section{Conclusions}

Based on our experiences, the majority of HCV-infected patients undergoing treatment with DAA agents can be easily monitored by non-specialist healthcare providers, addressing an important manpower issue. Therefore, access to care and treatment with DAA-based antivirals is not limited by the shortage of healthcare providers with expertise in treatment of HCV infection. Training for healthcare providers can be easily conducted by a hepatologist in-person or by telemedicine. The downstream benefits of task-shifting include higher awareness at the primary care level to screen for HCV infection and, therefore, increase the likelihood of early diagnosis and pre-emptive treatment of $\mathrm{CHC}$, which may be cost-effective in the long term.

While this review focuses on the domestic US implications of task-shifting for the management of HCV antiviral therapy, this treatment model may be applied on a global scale, particularly in under-resourced areas. ${ }^{20}$ It is important to ensure that task-shifting to nursing staff in the US occurs with appropriate oversight by a physician. Furthermore, we emphasize the importance of abiding by rules and regulations set by local, state and federal authorities in regards to maintaining the scope of practice for all non-specialist healthcare 
nursing staff. The Nurse Practice Act, for example, requires LVNs or LPNs to practice under the auspices of a registered nurse or a physician, and our task-shifting treatment model remains compliant with the current legislation. And, while the task-shifting treatment model traditionally utilizes nonspecialist healthcare providers such as nurses and advanced practice providers, primary care physicians may also fill the role to expand access further.

Although we believe our experiences can be generalized to other underserved settings, we recommend patients with cirrhosis and those who have undergone liver transplantation be treated with DAA-based therapy under close consultation with a transplant hepatology team at a tertiary care center. Finally, we believe these changes may improve HCV screening initiatives that have failed in the past and have led to late diagnoses of HCV, when liver transplant would be the only treatment option.

\section{Conflict of interest}

Dr. Aijaz Ahmed is a consultant and advisory board member for AbbVie Pharmaceuticals, Gilead Sciences, and Janssen Pharmaceuticals. Aijaz Ahmed has research funding/grant from Gilead Sciences. The others have no conflict of interest to declare.

\section{Author contributions}

Study concept and design (ERY, RBP, GC, CRJ, AA), acquisition of data (ERY, AA), analysis and interpretation of data (ERY, $A A)$, drafting of the initial and final manuscript (ERY, AA), critical revision of the manuscript (ERY, RBP, GC, CRJ, AA), and study supervision (AA).

\section{References}

[1] Stanaway JD, Flaxman AD, Naghavi M, Fitzmaurice C, Vos T, Abubakar I, et al. The global burden of viral hepatitis from 1990 to 2013: findings from the Global Burden of Disease Study 2013. Lancet 2016;388:1081-1088. doi: 10.1016/S0140-6736(16)30579-7.

[2] Smith DB, Bukh J, Kuiken C, Muerhoff AS, Rice CM, Stapleton JT, et al. Expanded classification of hepatitis $C$ virus into 7 genotypes and 67 subtypes: updated criteria and genotype assignment web resource. Hepatology 2014;59:318-327. doi: 10.1002/hep.26744.

[3] Ampuero J, Romero-Gómez M, Reddy KR. Review article: HCV genotype 3 the new treatment challenge. Aliment Pharmacol Ther 2014;39:686-698. doi: $10.1111 /$ apt.12646.
[4] Center for Disease Control and Prevention. Viral hepatitis - statistics \& surveillance. Available from: https://www.cdc.gov/hepatitis/statistics/, accessed October 4, 2016.

[5] Smith BD, Morgan RL, Beckett GA, Falck-Ytter Y, Holtzman D, Teo CG, et al. Recommendations for the identification of chronic hepatitis $C$ virus infection among persons born during 1945-1965. MMWR Recomm Rep 2012;61: $1-32$.

[6] Wong RJ, Aguilar M, Cheung R, Perumpail RB, Harrison SA, Younossi ZM, et al. Nonalcoholic steatohepatitis is the second leading etiology of liver disease among adults awaiting liver transplantation in the United States. Gastroenterology 2015;148:547-555. doi: 10.1053/j.gastro.2014.11.039.

[7] Spiegel BM, Younossi ZM, Hays RD, Revicki D, Robbins S, Kanwal F. Impact of hepatitis $C$ on health related quality of life: a systematic review and quantitative assessment. Hepatology 2005;41:790-800. doi: 10.1002/hep.20659.

[8] Lee MH, Yang HI, Lu SN, Jen CL, You SL, Wang LY, et al. Chronic hepatitis C virus infection increases mortality from hepatic and extrahepatic diseases: a community-based long-term prospective study. J Infect Dis 2012;206: 469-477. doi: 10.1093/infdis/jis385.

[9] Wise M, Bialek S, Finelli L, Bell BP, Sorvillo F. Changing trends in hepatitis C-related mortality in the United States, 1995-2004. Hepatology 2008;47: 1128-1135. doi: 10.1002/hep.22165.

[10] Reau N. HCV testing and linkage to care: Expanding access. Clinical Liver Disease 2014;4:31-34. doi: 10.1002/cld.376.

[11] Wirth S, Ribes-Koninckx C, Calzado MA, Bortolotti F, Zancan L, Jara P, et al. High sustained virologic response rates in children with chronic hepatitis $C$ receiving peginterferon alfa-2b plus ribavirin. J Hepatol 2010;52:501-507. doi: $10.1016 / j$.jhep.2010.01.016.

[12] Eisenman T. FDA approves Epclusa for treatment of chronic Hepatitis C virus infection. Available from https://www.fda.gov/NewsEvents/Newsroom/PressAnnouncements/ucm508915.htm, accessed June 28, 2016.

[13] Kredo T, Adeniyi FB, Bateganya M, Pienaar ED. Task shifting from doctors to non-doctors for initiation and maintenance of antiretroviral therapy. Cochrane Database Syst Rev 2014;(7):CD007331. doi: 10.1002/14651858. CD007331.pub3.

[14] Kredo T, Ford N, Adeniyi FB, Garner P. Decentralising HIV treatment in lowerand middle-income countries. Cochrane Database Syst Rev 2013;(6): CD009987. doi: 10.1002/14651858.CD009987.pub2.

[15] Jayasekera CR, Arora S, Ahmed A. Hepatitis C treatment delivery mandates optimizing available health care human resources: a case for task shifting. JAMA 2016;315:1947-1948. doi: 10.1001/jama.2016.1993.

[16] Arora S, Thornton K, Jenkusky SM, Parish B, Scaletti JV. Project ECHO: linking university specialists with rural and prison-based clinicians to improve care for people with chronic hepatitis C in New Mexico. Public Health Rep 2007;122 Suppl 2:74-77. doi: 10.1177/00333549071220S214.

[17] Arora S, Thornton K, Murata G, Deming P, Kalishman S, Dion D, et al. Outcomes of treatment for hepatitis $C$ virus infection by primary care providers. N Engl J Med 2011;364:2199-2207. doi: 10.1056/NEJMoa1009370.

[18] Tahan V, Almashhrawi A, Mutrux R, Ibdah JA. Show Me ECHO-Hepatitis C: A telemedicine mentoring program for patients with hepatitis $C$ in underserved and rural areas in Missouri as a model in developing countries. Turk J Gastroenterol 2015;26:447-449. doi: 10.5152/tjg.2015.159000.

[19] Lim SG, Aghemo A, Chen PJ, Dan YY, Gane E, Gani R, et al. Management of hepatitis $C$ virus infection in the Asia-Pacific region: an update. Lancet Gastroenterol Hepatol 2017:2:52-62. doi: 10.1016/S2468-1253(16)30080-2.

[20] Yoo ER, Perumpail RB, Cholankeril G, Jayasekera CR, Ahmed A. Task-shifting A practical strategy to improve the global access to treatment for chronic hepatitis C. Int J Nurs Stud 2016;62:168-169. doi: 10.1016/j.ijnurstu. 2016.07.023. 